\title{
Dynamical correction to linear Kohn-Sham conductances from static density functional theory
}

\author{
S. $\operatorname{Kurth}^{1,2,3}$ and G. Stefanucci ${ }^{4,5,3}$ \\ ${ }^{1}$ Nano-Bio Spectroscopy Group, Departamento de Física de Materiales, \\ Universidad del País Vasco UPV/EHU, Centro Física de Materiales CSIC-UPV/EHU, \\ Avenida Tolosa 72, E-20018 San Sebastián, Spain \\ ${ }^{2}$ IKERBASQUE, Basque Foundation for Science, E-48011 Bilbao, Spain \\ ${ }^{3}$ European Theoretical Spectroscopy Facility (ETSF) \\ ${ }^{4}$ Dipartimento di Fisica, Università di Roma Tor Vergata, \\ Via della Ricerca Scientifica 1, 00133 Rome, Italy \\ ${ }^{5}$ INFN, Laboratori Nazionali di Frascati, Via E. Fermi 40, 00044 Frascati, Italy
}

\begin{abstract}
For molecules weakly coupled to leads the exact linear Kohn-Sham (KS) conductance can be orders of magnitude larger than the true linear conductance due to the lack of dynamical exchangecorrelation (xc) corrections. In this work we show how to incorporate dynamical effects in KS transport calculations. The only quantity needed is the static xc potential in the molecular junction. Our scheme provides a comprehensive description of Coulomb blockade without breaking the spin symmetry. This is explicitly demonstrated in single-wall nanotubes where the corrected conductance is in good agreement with experimental data whereas the KS conductance fails dramatically.
\end{abstract}

PACS numbers: 05.60.Gg, 31.15.ee, 71.15.Mb, 73.63.-b

The active field of molecular electronics [1] remains a challenge for $a b$ initio methods. Density Functional Theory (DFT) is at present the only viable route for an atomistic description of complex molecular junctions. Nevertheless, DFT transport calculations still suffer from some practical difficulties. The fundamental sources of error in the linear conductance are the DFT exchange-correlation (xc) potential used to determine the Kohn-Sham (KS) conductance $G_{s}$ and the dynamical xc correction [2 5] predicted by time-dependent (TD) DFT [ 6] (see Eq. (9) below). Assessing their importance and mutual interplay is especially thorny in weakly coupled molecules where level alignment and charging effects play a prominent role. Toher et al. 7] and Koentopp et al. [4] showed that $G_{s}$ evaluated with an accurate, and hence discontinuous [8], xc potential is suppressed, thus capturing the Coulomb blockade (CB) effect at even electron numbers $N$ (closed shell). This may suggest the dynamical xc correction to be small. However, at odd $N$ (open shell) $G_{s}$ can be orders of magnitude larger than the true conductance $G$, even when the exact xc potential is employed [10]. A satisfactory "DFT explanation" of $\mathrm{CB}$ is, therefore, currently missing. In this Letter we provide a comprehensive picture of $\mathrm{CB}$, valid for all $N$ without breaking the spin symmetry. The key ingredient is the dynamical xc correction which, remarkably, can be expressed exclusively in terms of static DFT quantities. We propose a practical scheme to calculate $G$ and demonstrate its validity by comparison with recent experiments on single-wall nanotubes.

At zero temperature and when transport is dominated by a single resonance (open shell) $G_{s}=G$ due to the Friedel sum rule [9]. In this regime $G$ exhibits a Kondo plateau (no CB peaks) and the discontinuity is essential

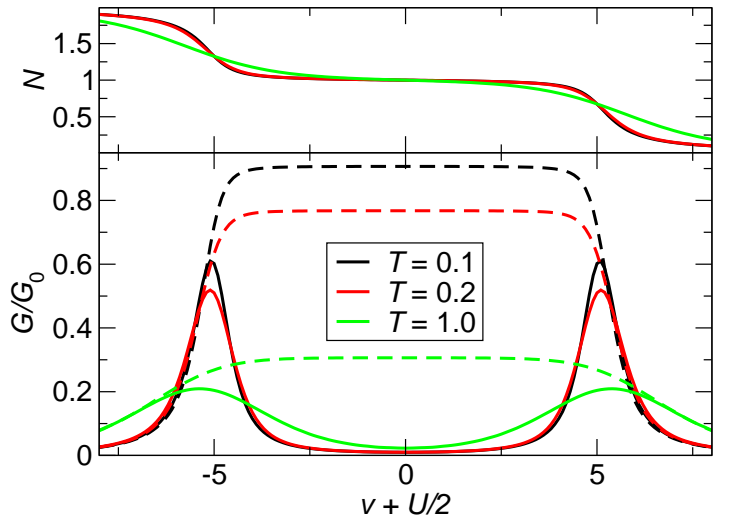

FIG. 1. Top: Electron number $N$ versus gate in MB and DFT (indistinguishable) for a single level coupled to featureless leads with $U=10, \mu=0$ at various temperatures $T$ (all energies in units of $\gamma$ ). For these parameters $T_{\mathrm{K}}=\sqrt{U \gamma} \exp \left(-\frac{\pi U}{8 \gamma}\right) \simeq 0.06$. Bottom: $G$ from Eq. (2) (solid) and $G_{s}$ from Eq. (4) (dashed) in units of $G_{0}=2 e^{2} / h$.

for $G_{s}$ to reproduce the plateau [10 12]. At temperatures higher than the Kondo temperature $T_{\mathrm{K}}$, the exact discontinuous xc potential gives instead a $G_{s} \gg G[10]$. To understand this discrepancy we model the resonance with a single level (HOMO or LUMO) of energy $v$ and Coulomb repulsion $U$ coupled to left $(L)$ and right $(R)$ featureless leads contributing $\gamma=\gamma_{L}+\gamma_{R}$ to the broadening of the spectral peaks. Given the Many-Body (MB) spectral function $A(\omega)$ the number of electrons is

$$
N=2 \int f(\omega) A(\omega), \quad \int \equiv \int \frac{d \omega}{2 \pi}
$$


whereas the linear (zero-bias) conductance reads

$$
G=-2 \frac{\gamma_{L} \gamma_{R}}{\gamma} \int f^{\prime}(\omega) A(\omega)
$$

with the Fermi function $f(\omega)=1 /\left(e^{\beta(\omega-\mu)}+1\right)$ at inverse temperature $\beta=1 / T$ and chemical potential $\mu$. At $T \gg$ $T_{\mathrm{K}}$ the Abrikosov-Suhl (AS) resonance is suppressed and the spectral function is well represented by [13]

$$
A(\omega)=n L_{\gamma}(\omega-v-U)+(1-n) L_{\gamma}(\omega-v)
$$

where $n=N / 2$ and $L_{\gamma}(\omega)=\gamma /\left(\omega^{2}+\gamma^{2} / 4\right)$. For the $\mathrm{KS}$ system the spectral function is $A_{s}(\omega)=L_{\gamma}(\omega-$ $\left.v-v_{\mathrm{Hxc}}[N]\right)$. The Hartree-Xc (Hxc) potential $v_{\mathrm{Hxc}}$ is such that the number of electrons $N$ which solves $N=$ $2 \int f(\omega) A_{s}(\omega)$ is the same as in Eq. (11). We obtain $v_{\mathrm{Hxc}}$ by reverse engineering and find that, as function of $N$, it has the shape of a smeared step function (not shown). The smearing is due to the level broadening $\gamma$ induced by contacting the level to the leads and develops into a true discontinuity (at $N=1$ ) only in the limit $\gamma \rightarrow 0$, as it should [11, 12]. This $v_{\mathrm{Hxc}}$ is then used to calculate the KS conductance from

$$
G_{s}=-2 \frac{\gamma_{L} \gamma_{R}}{\gamma} \int f^{\prime}(\omega) A_{s}(\omega)
$$

Despite the fact that the $\mathrm{MB}$ and DFT $N-v$ curves are identical, see Fig. 1 top, the CB peaks present in $G$ are completely absent in $G_{s}$, see Fig. 1 bottom. The physical situation discussed here is distinct from that of Ref. 7 where the discontinuity keeps the HOMO doubly occupied and the LUMO empty when gating the molecule (closed shell). The discontinuity correctly suppresses $G_{s}$ at even $N$ but has the opposite effect at odd $N$.

Dynamical xc effects: Open-shell molecules in the CB regime are probably the most striking example of the inadequacy of standard DFT transport calculations. Below we derive an exact formula for $G$ in terms of TDDFT quantities. We take the leads as two jellia (the argument can be generalized to more realistic leads) and choose $z$ as the longitudinal coordinate so that $z \rightarrow-\infty$ is in the left lead, $\alpha=L$, whereas $z \rightarrow \infty$ is in the right lead, $\alpha=R$. Let $\delta V^{\alpha}$ be the variation in the classical potential (external plus Hartree) of lead $\alpha$. This perturbation generates a current [2, 4]

$$
\delta I=\left(\delta V^{R}-\delta V^{L}+\delta V_{\mathrm{xc}}^{R}-\delta V_{\mathrm{xc}}^{L}\right) G_{s}
$$

where $\delta V_{\mathrm{xc}}^{\alpha}=\lim _{t \rightarrow \infty} \lim _{z \rightarrow s_{\alpha} \infty} \delta v_{\mathrm{xc}}(\mathbf{r}, t), s_{R / L}= \pm$, is the asymptotic value of the variation of the xc potential $\delta v_{\mathrm{xc}}$. From linear-response TDDFT

$$
\delta V_{\mathrm{xc}}^{\alpha}=\int d t^{\prime} d \mathbf{r}^{\prime} \lim _{t \rightarrow \infty} \lim _{z \rightarrow s_{\alpha} \infty} f_{\mathrm{xc}}\left(\mathbf{r}, \mathbf{r}^{\prime} ; t-t^{\prime}\right) \delta n\left(\mathbf{r}^{\prime}, t^{\prime}\right)
$$

where $f_{\mathrm{xc}}$ is the TDDFT kernel and $\delta n(\mathbf{r}, t)$ is the density variation. The assumption of a steady state implies that $f_{\mathrm{xc}} \rightarrow 0$ for $\left|t-t^{\prime}\right| \rightarrow \infty$ and $\delta n(\mathbf{r}, t \rightarrow \infty)=s_{\alpha} \delta n$ for $\mathbf{r}$ in lead $\alpha$. In Eq. (6) the contribution of the molecular region to the spatial integral is negligible in the thermodynamic limit. If we define

$$
f_{\mathrm{xc}}^{\alpha \beta}=\int d t^{\prime} \int_{\text {lead } \beta} d \mathbf{r}^{\prime} \lim _{z \rightarrow s_{\alpha} \infty} f_{\mathrm{xc}}\left(\mathbf{r}, \mathbf{r}^{\prime} ; t^{\prime}\right)
$$

then $\delta V_{\mathrm{xc}}^{\alpha}=\sum_{\beta=L, R} f_{\mathrm{xc}}^{\alpha \beta} s_{\beta} \delta n$. We emphasize that $f_{\mathrm{xc}}^{\alpha \beta}$ is not the static DFT kernel since the limit $t \rightarrow \infty$ is taken after the limit $|z| \rightarrow \infty$ and these two limits, in general, do not commute [14]. This implies that we cannot model $f_{\mathrm{xc}}^{\alpha \beta}$ by performing DFT calculations on leads of finite length. Inserting the expression for $\delta V_{\mathrm{xc}}^{\alpha}$ into Eq. (5) we find $\delta I=\left(\delta V^{L}-\delta V^{R}\right) G_{s}-\Phi G_{s} \delta n$ where

$$
\Phi \equiv f_{\mathrm{xc}}^{R L}+f_{\mathrm{xc}}^{L R}-f_{\mathrm{xc}}^{R R}-f_{\mathrm{xc}}^{L L} .
$$

The expression for $\delta I$ is correctly gauge invariant. The kernel $f_{\mathrm{xc}}$ is defined up to the addition of an arbitrary function $q(\mathbf{r})+q\left(\mathbf{r}^{\prime}\right)$ [15] and $\Phi$ is invariant under this transformation. In conclusion

$$
G \equiv \frac{\delta I}{\left(\delta V^{R}-\delta V^{L}\right)}=\frac{G_{s}}{1+\chi \Phi G_{s}} .
$$

The quantity $\chi \equiv \delta n / \delta I \simeq 1 /\left(v_{F} \sigma\right)$ with $v_{F}$ the Fermi velocity and $\sigma$ the cross section of the leads [16. In the following we define $\chi \Phi G_{s}$ as the dynamical xc correction since $\Phi$ is expressed in terms of the TDDFT kernel.

Approximations to $\Phi$ : To gain some insight into the density dependence of $\chi \Phi$ we consider again the single level model. For $N \neq 1$ and $T<\gamma$ the real and KS systems respond similarly and consequently $G \simeq G_{s}$. On the other hand for $N=1$ we have $G \simeq 0$ whereas $G_{s} \simeq G_{0}=2 e^{2} / h$ the quantum of conductance. Therefore $\chi \Phi$ is small for $N \neq 1$ and large for $N=1$. Interestingly the quantity $\partial v_{\mathrm{Hxc}} / \partial N$ behaves similarly. Is there any relation between $\chi \Phi$ and $\partial v_{\mathrm{Hxc}} / \partial N$ ? If so this relation would simplify enormously the problem of estimating the dynamical xc correction since $\partial v_{\mathrm{Hxc}} / \partial N$ can be calculated from static DFT. In the following we show that in the CB regime this relation does actually exist.

Consider the system in equilibrium. Using Eq. (1) the compressibility $\kappa=\partial N / \partial \mu$ can be written as $\kappa=\frac{\gamma}{\gamma_{L} \gamma_{R}} G+2 \int f(\omega) \partial A(\omega) / \partial \mu$, where we identified the conductance $G$ of Eq. (2). If we define the quantity $R \equiv-2 \int f(\omega) \partial A(\omega) / \partial N$ then $\kappa=\frac{\gamma}{\gamma_{L} \gamma_{R}} G /(1+R)$. As the MB and DFT densities are the same, the MB and DFT compressibilities are the same too. Hence $\kappa=\frac{\gamma}{\gamma_{L} \gamma_{R}} G_{s}+2 \int f(\omega) \partial A_{s}(\omega) / \partial \mu$, where we identified the KS conductance $G_{s}$ of Eq. (4). The KS spectral function depends on $\mu$ through $N$, and the dependence on $N$ is all contained in $v_{\mathrm{Hxc}}$. Since $\frac{\partial A_{s}}{\partial v_{\mathrm{Hxc}}}=-\frac{\partial A_{s}}{\partial \omega}$ (see definition of $A_{s}$ below Eq. (3) ) we have $\frac{\partial A_{s}}{\partial \mu}=-\frac{\partial A_{s}}{\partial \omega} \frac{\partial v_{\mathrm{Hxc}}}{\partial N} \frac{\partial N}{\partial \mu}$. Using this result under the integral, solving for $\kappa$ and 
equating the MB and DFT expressions one easily obtains

$$
\frac{G}{G_{s}}=\frac{1+R}{1+\frac{\gamma}{\gamma_{L} \gamma_{R}} G_{s} \frac{\partial v_{\mathrm{Hxc}}}{\partial N}} .
$$

No approximations have been made so far. Let us study the dependence of $R$ on temperature.

We first consider the low temperature case. For simplicity we take $\gamma_{L}=\gamma_{R}$ and set $v=-U / 2$ at the particlehole (ph) symmetric point (half-filling). At zero temperature $G=G_{s}=G_{0}$ and hence $R=R_{0} \equiv \frac{4 G_{0}}{\gamma} \frac{\partial v_{\mathrm{Hxc}}}{\partial N}$ [17]. For temperatures $T>T_{\mathrm{K}}$ the AS resonance broadens and its height decreases as $h\left(T / T_{\mathrm{K}}\right)$ where $h$ is a universal function which approaches zero at high $T$ 21]. This means that $R \simeq h\left(T / T_{K}\right) R_{0}$ remains large until the AS resonance disappears. No simple relation between $\Phi$ and $\partial v_{\mathrm{Hxc}} / \partial N$ exists when Kondo correlations are present.

At temperatures $T \gg T_{\mathrm{K}}$ thermal fluctuations destroy the Kondo effect and the MB spectral function is well approximated by Eq. (3)). Therefore $R(v)=I(v)-I(v+$ $U)$ where $I(E) \equiv \int f(\omega) L_{\gamma}(\omega-E)$. We can derive a more convenient expression for $R$ by inserting Eq. (3) into Eq. (1) to find

$$
N=\frac{2 I(v)}{1+I(v)-I(v+U)}
$$

and hence $1+R=2 I(v) / N$. Unfortunately $I(v)$ is not an explicit function of $N$ due to the implicit dependence of $v=v[N]$. However, for $v<\mu$, or equivalently for $N<1$, we have $I(v+U) \ll 1$. Thus for $N<1$ we can write $N \simeq$ $2 I(v) /(1+I(v))$ from which we infer $I(v) \simeq N /(2-N)$. Using ph symmetry we therefore approximate $R$ by the explicit function $1+R=2 /(1+|\delta N|)$ where $\delta N=N-1$. Inserting this into Eq. (10) we deduce the main result of this Letter

$$
\frac{G}{G_{s}}=\frac{2}{1+|\delta N|} \frac{1}{1+\frac{\gamma}{\gamma_{L} \gamma_{R}} G_{s} \frac{\partial v_{\mathrm{Hxc}}}{\partial N}} .
$$

Equation (12) provides a simple and implementable formula to correct the KS conductance. In fact, the dynamical xc correction of Eq. (9) is entirely expressed in terms of static DFT quantities. Moreover, whereas $\Phi$ involves the TDDFT kernel with coordinates in the leads the correction in Eq. (12) involves only the DFT $v_{\mathrm{Hxc}}$ in the molecular junction. The accuracy of Eq. (12) is examined in Fig. 2, and benchmarked against the MB conductance of Eq. (2). Even though the approximate $R$ is not on top of the exact one, see inset, the agreement between the two conductances is extremely good. The position, width and height of the peaks as well as the decay for large $|v|$ are all well reproduced. Most importantly the plateau of $G_{s}$, see Fig. 1. is completely gone.

Application to physical systems: In real molecules $v_{\mathrm{Hxc}}$ is a $\mathbf{r}$-dependent functional of the density. We

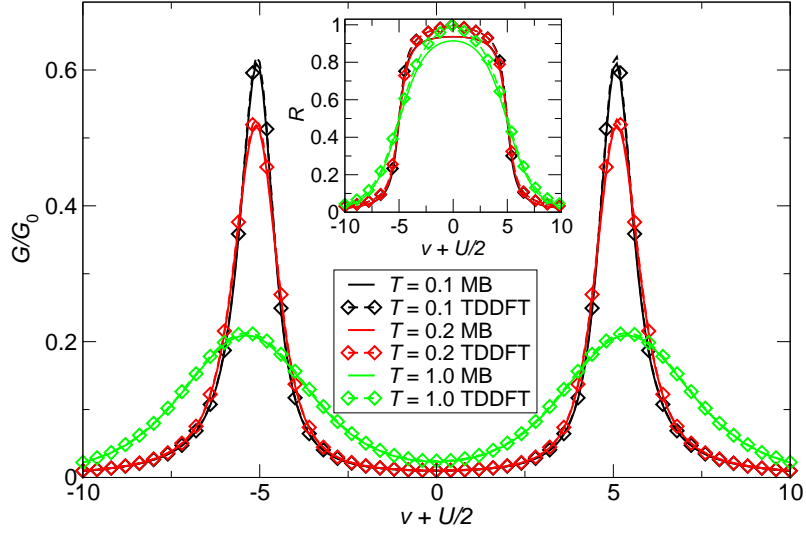

FIG. 2. Linear conductance from Eq. (2) using the spectral function Eq. (3) (MB, solid) and from Eq. (12) (TDDFT, dashed). The inset shows a comparison between the exact and the approximate $R$. Same parameters as in Fig. 1]

write $v_{\mathrm{Hxc}}(\mathbf{r})=\delta v_{\mathrm{Hxc}}(\mathbf{r})+\bar{v}_{\mathrm{Hxc}}$ as the sum of a functional $\delta v_{\mathrm{Hxc}}$ with a weak dependence on $N=\int_{V} d \mathbf{r} n(\mathbf{r})$ and a spatially uniform part $\bar{v}_{\mathrm{Hxc}}=\frac{1}{V} \int_{V} d \mathbf{r} v_{\mathrm{Hxc}}(\mathbf{r})$, where the integral is over the volume $V$ of the molecular junction. For weakly coupled molecules $\bar{v}_{\mathrm{Hxc}}$ exhibits sharp steps as function of $N$ when $N$ crosses an integer. If we are at resonance and spin fluctuations are suppressed (no Kondo effect) then the KS conductance must be corrected according to Eq. (12) in which $\partial v_{\mathrm{Hxc}} / \partial N \rightarrow \partial \bar{v}_{\mathrm{xc}} / \partial N$. Below we argue that this correction applies out-of-resonance too. Let $\mu$ be in the HOMO-LUMO gap and consider a two-level system with $\Gamma_{\alpha}$ the $2 \times 2$ broadening matrix. For general $\Gamma_{\alpha}$ no simple analytic relation between $G$ and $N$ exists. However if $\Gamma_{\alpha, m l}=\left(\gamma_{\alpha} / 2\right) \delta_{m l}$ then $N=2 \int f(\omega) \operatorname{Tr}[A(\omega)]$ and $G=-2 \frac{\gamma_{L} \gamma_{R}}{\gamma} \int f^{\prime}(\omega) \operatorname{Tr}[A(\omega)]$. Discarding the dependence of $\delta v_{\mathrm{Hxc}}$ on $\mu$ (which is weak by definition) we can go through the same steps of the single-level derivation and find again Eq. (12). It is therefore reasonable to expect that the KS conductance should be corrected even out-of-resonance (closed shell) and that this correction should be proportional to $G_{s} \partial \bar{v}_{\mathrm{Hxc}} / \partial N$.

We here propose a practical scheme to calculate $G$ from DFT. Given the KS Hamiltonian matrix $h_{\mathrm{KS}, m l}=\delta_{m l} \epsilon_{l}$ and the broadening matrices $\Gamma_{\alpha, m l}$ we determine the density and $G_{s}$ in the usual manner. $G$ is then obtained from Eq. (12) where $\delta N$ is the deviation of $(N-\operatorname{Int}[N])$ from 1 whereas $\gamma_{\alpha}=\gamma_{\alpha}(N)=\Gamma_{\alpha, H H}$ if $\mu \simeq \epsilon_{H}$ (resonance, open shell) and $\gamma_{\alpha}(N)=\frac{1}{2}\left(\Gamma_{\alpha, H H}+\Gamma_{\alpha, L L}\right)$ if $\mu \simeq \frac{1}{2}\left(\epsilon_{L}+\epsilon_{H}\right)$ is in the HOMO-LUMO gap (out-ofresonance, closed shell). One could improve the approximation to $\gamma_{\alpha}$ using different weights, but the qualitative features of the results are independent of these details.

To appreciate the decisive impact of the dynamical xc correction we consider two paradigmatic junctions in which $\delta v_{\text {Hxc }}$ can be discarded. For $\bar{v}_{\text {Hxc }}$ we choose a best 


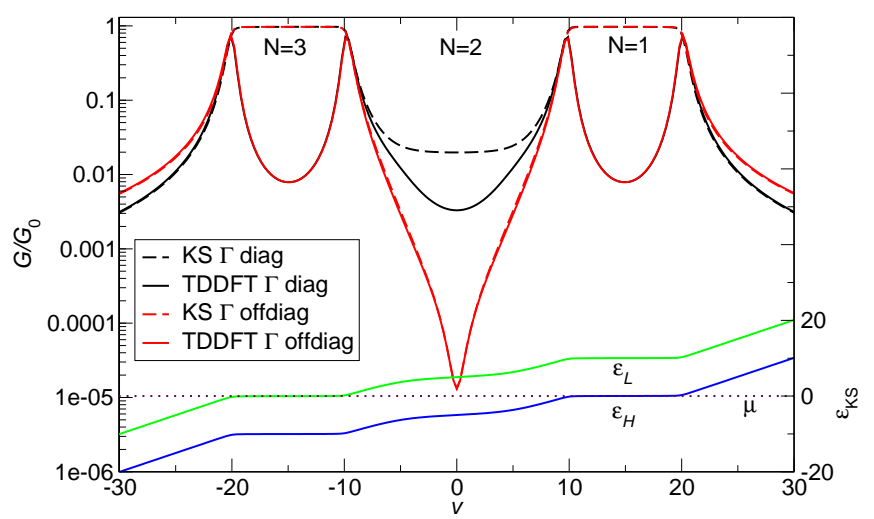

FIG. 3. Linear conductance from Eq. (4) (KS, dashed) and from Eq. (12) (TDDFT, solid) for the HOMO-LUMO model with diagonal and off-diagonal $\Gamma$-matrices (left axis) and KS energies $\epsilon_{H / L}=\epsilon_{0 H / L}+\bar{v}_{\mathrm{Hxc}}$ (right axis). The electron number $N$ for different ranges of $v$ is also indicated.

fit of the zero-temperature limit of the single-level Hxc potential, but now sum over all possible charged states of the molecule 22, i.e.,

$$
\bar{v}_{\mathrm{Hxc}}=\sum_{K} \frac{U(K)}{\pi} \arctan \left(\frac{N-K}{W(K)}\right) .
$$

The charging energies $U(N)$ are given by the xc part of the derivative discontinuity of the molecule with $N$ electrons [8]. For the widths we take $W(N)=$ $0.16 \gamma(N) / U(N)$ which is consistent with Ref. 19

HOMO-LUMO model: We study a two-level system with 2 electrons in the $\mathrm{HOMO}$ in the charge neutral state. Let $\epsilon_{0 H}=-\epsilon_{0 L}=-\epsilon_{0}<0$ be the noninteracting singleparticle energies,

$$
\Gamma_{L}=\Gamma_{R}=\frac{\gamma}{2}\left(\begin{array}{ll}
1 & 1 \\
1 & 1
\end{array}\right)
$$

and $U(N)=U$ independent of $N$. We solve the selfconsistent equation for the density with $U=10, \epsilon_{0}=5$, $\mu=0$ and $\beta=10$ (all energies in units of $\gamma$ ). $G_{s}$ and $G$ from Eq. (12) are shown in Fig. 3 (left axis). As expected the discontinuity of $v_{\mathrm{Hxc}}$ opens a gap in $G_{s}$ for even $N$, in agreement with the results of Ref. 7 . Here the dynamical xc correction only weakly affects $G_{s}$ since $\partial \bar{v}_{\mathrm{Hxc}} / \partial N$ is multiplied by $G_{s} \ll 1$. For odd $N$ the KS conductance exhibits a Kondo plateau due to the pinning of the KS level to $\mu$, see right axis. This is the regime previously discussed and no $\mathrm{CB}$ is observed. The dynamical xc correction remedies this serious deficiency by correctly suppressing the plateau. The results remain essentially unaltered if the off-diagonal matrix elements of $\Gamma_{\alpha}$ are discarded [23].

$S W N T$ : Experimental evidence of CB oscillations has recently been reported in metallic single-wall nanotubes

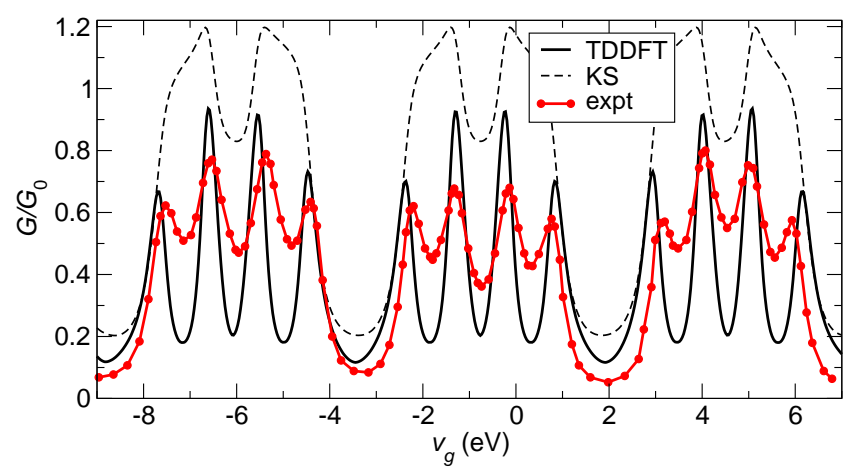

FIG. 4. Linear KS and TDDFT conductance (Eq. (12) for a SWNT quantum dot in comparison to experimental conductance from Ref. 24, as function of gate voltage $v_{g}$.

(SWNT) quantum dots 24 26]. We now analyze the performance of Eq. (12) in these systems. The finite length of the SWNT causes a level quantization of the twofold degenerate bands. Since the wavevector is a good quantum number our approximation $\delta v_{\mathrm{Hxc}}=0$ is justified. For a SWNT quantum dot the constant interaction model 27] has been refined by Oreg et al. 28] to account for the observed fourfold periodicity in the electron addition energy. We constructed the KS Hamiltonian corresponding to this model and approximated the broadening matrix as $\Gamma_{\alpha, m l}=(\gamma / 2) \delta_{m l}$ [29] (no visible interference 23] from experiment). In Fig. 4 we compare the KS, TDDFT and experimental conductance versus the gate voltage $v_{g}$. We clearly see that the conductance of Eq. (12) correctly exhibits the fourfold periodicity and represents a considerable improvement over $G_{s}$ which, instead, shows two deformed Kondo plateaus per period. The qualitative behavior of $G$ and $G_{s}$ does not change by varying the parameters within a reasonable range around the average values reported in Ref. 24 .

In conclusion we proposed a practical scheme to correct $\mathrm{KS}$ conductances. We highlighted the role of the discontinuity not only for an accurate $G_{s}$ but also for an accurate dynamical xc correction to $G$. Approaches to generate discontinuous xc potentials are emerging both in the static 30] and dynamical [31] case. Our theory provides a coherent picture of CB within (TD)DFT without breaking the spin symmetry. By application to two different molecular junctions we further showed that the dynamical xc correction always reduces $G_{s}$, thus contributing to close the gap between theoretical predictions and experimental measurements.

S.K. acknowledges funding by the "Grupos Consolidados UPV/EHU del Gobierno Vasco" grant No. IT57813. G.S. acknowledges funding by MIUR FIRB grant No. RBFR12SW0J. We acknowledge support through travel grants (Psi-K2 4665 and 3962 (G.S.), and Psi-K2 5332 (S.K.)) of the European Science Foundation (ESF). 
[1] G. Cuniberti, G. Fagas and. K. Richter, Introducing Molecular Electronics, (Springer, Heidelberg, 2005); J.C. Cuevas and E. Scheer, Molecular Electronics: An Introduction to Theory and Experiment, (World Scientific, London, 2010).

[2] G. Stefanucci and C.-O. Almbladh, Phys. Rev. B 69, 195318 (2004); Europhys. Lett. 67, 14 (2004).

[3] N. Sai, M. Zwolak, G. Vignale, and M. Di Ventra, Phys. Rev. Lett. 94, 186810 (2005).

[4] M. Koentopp, K. Burke, and F. Evers, Phys. Rev. B 73, 121403 (2006).

[5] G. Vignale and M. Di Ventra, Phys. Rev. B 79, 014201 (2009).

[6] E. Runge and E. K. U. Gross, Phys. Rev. Lett. 52, 997 (1984).

[7] C. Toher et al., Phys. Rev. Lett. 95, 146402 (2005).

[8] J. P. Perdew et al., Phys. Rev. Lett. 49, 1691 (1982).

[9] H. Mera et al., Phys. Rev. B 81, 035110 (2010); H. Mera and Y. M. Niquet, Phys. Rev. Lett. 105, 216408 (2010).

[10] G. Stefanucci and S. Kurth, Phys. Rev. Lett. 107, 216401 (2011).

[11] J. P. Bergfield, et al., Phys. Rev. Lett. 108, 066801 (2012).

[12] P. Tröster, P Schmitteckert and F. Evers, Phys. Rev. B 85, 115409 (2012)

[13] See, e.g., H. Haug and A.-P. Jauho, Quantum Kinetics in Transport and Optics of Semiconductors (Springer, 2008).

[14] The static DFT kernel is the response of the equilibrium $v_{\mathrm{xc}}$ to a density variation and, deep inside the leads, is determined by the condition of charge neutrality alone.

[15] This follows from the fact that $\delta v_{\mathrm{xc}}(\mathbf{r}, t \rightarrow \infty)$ is defined up to the addition of an arbitary constant $C$, see also M. Hellgren and E. K. U. Gross, Phys. Rev. A 85, 022514 (2012).

[16] A.M. Uimonen et al., Phys. Rev. B 84, 115103 (2011).

[17] The compressibility is weakly dependent on temperature up to $T \simeq \gamma$ [18]. Therefore the $v_{\mathrm{Hxc}}$ obtained by reverse engineering is a good approximation even for $T=0$. At the ph symmetric point $(N=1)$ this approximation gives $\partial v_{\mathrm{Hxc}} / \partial N \sim U^{2} / \gamma[19]$. At $T=0$ the MB spectral function for $\omega \simeq \mu$ is dominated by the $\mathrm{AS}$ resonance $A(\omega \simeq \mu) \simeq \frac{4 T_{\mathrm{K}}}{\gamma} L_{T_{\mathrm{K}}}(\omega-\mu)$ and thus $\int f(\omega) \frac{\partial A(\omega)}{\partial \mu} \simeq-\frac{2}{\pi \gamma}$. For the compressibility we have from the Bethe-Ansatz 20] $\kappa=(8 \gamma) /\left(\pi U^{2}\right)[1+\mathcal{O}(\gamma / U)]$ from which it follows that also $R$ goes like $(U / \gamma)^{2}$.

[18] I. Maruyama, N. Shibata and K. N. Ueda, J. Phys. Soc. Jpn 73, 434 (2004).

[19] F. Evers and P. Schmitteckert, Phys. Chem. Chem. Phys. 13, 14417 (2011).

[20] P. B. Wiegmann and A. M. Tsvelick, J. Phys. C: Solid State Phys. 16, 2281 (1983)

[21] T. A. Costi, Phys. Rev. Lett. 85, 1504 (2000).

[22] E. Perfetto and G. Stefanucci, Phys Rev B 86, 081409 (2012)

[23] This amounts to neglecting interference effects, see G. Stefanucci et al., Phys. Rev. B 79, 073406 (2009); T. Markussen, R. Stadler and K. S. Thygesen, Nano Lett. 10, 4260 (2010); J. P. Bergfield et al., Nano Lett. 11, 2759 (2011).
[24] W. Liang, M. Bockrath and H. Park, Phys. Rev. Lett. 88, $126801(2002)$

[25] M. R. Buitelaar et al., Phys. Rev. Lett. 88, 156801 (2002).

[26] S. Sapmaz et al., Phys. Rev. B 71, 153402 (2005).

[27] L. P. Kouwenhoven, D. G. Austing, and S. Tarucha, Rep. Prog. Phys. 64, 701 (2001).

[28] Y. Oreg, K. Byczuk and B. I. Halperin, Phys. Rev. Lett. 85, 365 (2000).

[29] see Supplemental Material for details on the implementation.

[30] N. A. Lima et al., Europhys. Lett. 60, 601 (2002); N. A. Lima et al., Phys. Rev. Lett. 90, 146402 (2003); P. MoriSanchez, A. J. Cohen, and W. T. Yang, Phys. Rev. Lett. 102, 066403 (2009); F. Malet and P Gori-Giorgi, Phys Rev. Lett. 109, 246402 (2012); X. Gao et al., Phys. Rev. B 86, 235139 (2012); J. Lorenzana, Z.-J. Ying, and V. Brosco, Phys. Rev. B 86, 075131 (2012); E. Kraisler and L. Kronik, Phys. Rev. Lett. 110, 126403 (2013).

[31] C. Verdozzi, Phys. Rev. Lett. 101, 166401 (2008); S. Kurth et al., Phys. Rev. Lett. 104, 236801 (2010); D. Hofmann and S. Kümmel, Phys. Rev. B 86, 201109(R) (2012); P. Elliott et al., Phys. Rev. Lett. 109, 266404 (2012); S.E.B. Nielsen, M. Ruggenthaler and R. van Leeuwen, EPL 101, 33001 (2013). 


\section{Supplemental Material}

In this Supplemental Material we refer to the paper as I. The many-body Hamiltonian of the SWNT quantum dot was proposed by Oreg et al. (PRL 85, 365 (2000)) and reads

$$
H=\sum_{l \nu \sigma} \epsilon_{0 l \nu} n_{l \nu \sigma}+\frac{1}{2} E_{C}\left(N-N_{0}\right)^{2}+\delta U \sum_{l \nu} n_{l \nu \uparrow} n_{l \nu \downarrow}+J N_{\uparrow} N_{\downarrow} .
$$

Here $\sigma$ is the spin index, $\nu=0,1$ is the band index and $l$ is the integer of the quantized quasi-momentum of the electrons. The Hamiltonian is written in terms of the occupation numbers $n_{l \nu \sigma}$ since $N_{\sigma} \equiv \sum_{l \nu} n_{l \nu \sigma}$ (total number of electrons with spin $\sigma$ ) and $N=N_{\uparrow}+N_{\downarrow}$ (total number of electrons). The finite length of the SWNT causes a finite subband mismatch $\delta$ so that the single-particle energies are

$$
\epsilon_{0 l \nu}= \begin{cases}l \Delta-\delta & \text { for } \quad \nu=0 \\ l \Delta & \text { for } \quad \nu=1\end{cases}
$$

with $\Delta$ the average level spacing. In Eq. (14) the parameter $E_{C}$ is the charging energy $\left(N_{0}\right.$ is the number of electrons of the charge neutral SWNT quantum dot), $\delta U$ is the extra charging energy for two electrons in the same energy level and $J$ is the exchange energy between electrons of opposite spin. With the parameters of W. Liang et al. (PRL 88, 126801 (2002)) when an extra electron enters the nanotube it occupies the lowest available single-particle energy level.Thus the aufbau is the same as that of the noninteracting Hamiltonian. Accordingly the spin of the ground state is $0,1 / 2,0,1 / 2, \ldots$ for $0,1,2,3, \ldots$ extra electrons. Let $E(N)$ be the energy of the SWNT with $N$ extra electrons. Using Eq. (14) it is straightforward to obtain

$$
\begin{aligned}
& E(0)=0 \\
& E(1)=\Delta-\delta+\frac{1}{2} E_{C} \\
& E(2)=2 \Delta-2 \delta+\frac{1}{2} 4 E_{C}+\delta U+J, \\
& E(3)=3 \Delta-2 \delta+\frac{1}{2} 9 E_{C}+\delta U+2 J,
\end{aligned}
$$

and so on. The function $E(N)$ with $N$ a real continuous variable has a discontinuous derivative at integers $N$ and the size of this discontinuity is given by $\Delta(N)=E(N+1)-2 E(N)+E(N-1)$, see Perdew et al. (PRL 49, 1691 (1982)). One finds

$$
\begin{aligned}
& \Delta(1)=E_{C}+\delta U+J, \\
& \Delta(2)=\delta+E_{C}-\delta U,
\end{aligned}
$$

and $\Delta(N)=\Delta(K)$ if $N-K=0 \bmod 2$ with $K=1,2$. We now turn to the KS system. The KS Hamiltonian of the SWNT has the general form

$$
H_{\mathrm{KS}}=\sum_{l \nu \sigma}\left(\epsilon_{0 l \nu}+v_{\mathrm{Hxc}, l \nu}[\{n\}]\right) n_{l \nu \sigma}
$$

The Hxc potential $v_{\mathrm{Hxc}, l \nu}$ has the property that for a given chemical potential the ground state occupations $n_{l \nu \sigma}$ of $H$ and of $H_{\mathrm{KS}}$ are identical. Since $H$ is diagonal in the occupation basis and follows the aufbau of the noninteracting Hamiltonian, we have that $\left.v_{\mathrm{Hxc}, l \nu}[\{n\}]\right)=\bar{v}_{\mathrm{Hxc}}[N]$ is uniform and depends only on the total number of particles $N$. Thus, for the SWNT the fluctuation $\delta v_{\mathrm{Hxc}}$ around the average $\bar{v}_{\mathrm{Hxc}}$ (these quantities have been introduced in "Application to physical systems" of I) is exactly zero. For an isolated SWNT the potential $\bar{v}_{\mathrm{Hxc}}[N]$ is discontinuous every time $N$ crosses an integer and the size of the step, $U$, is given by the xc part of the discontinuity $\Delta$. From Eqs. (17) we find

$$
\begin{aligned}
& U(1)=E_{C}+\delta U+J, \\
& U(2)=E_{C}-\delta U,
\end{aligned}
$$

and $U(N)=U(K)$ if $N-K=0 \bmod 2$ with $K=1,2$. The average values of these parameters can be found in W. Liang et al. (PRL 88, $126801(2002)$ ). Here we change them slightly to match the peak positions of the SWNT of 
length $\simeq 100 \mathrm{~nm}$ (all energies are in meV): $\Delta=9.2, \delta=2.27$, charging energy $E_{C}=2.485$, exchange energy $J=0.7$, extra charging energy for doubly occupied levels $\delta U=0.37$. The sharp steps of $\bar{v}_{\mathrm{Hxc}}$ are smeared when the SWNT is brought in contact with the leads. To find the functional form of $\bar{v}_{\mathrm{Hxc}}$ we observe that the exact smeared potential which produces the electron number of Fig. 1 of I (single level model) is well approximated by

$$
v_{\mathrm{Hxc}}[N]=\frac{U}{2}+\frac{U}{\pi} \arctan \left(\frac{N-1}{W}\right)
$$

where $W \simeq 0.16 \gamma / U$. Therefore a good approximation to the Hxc potential is [cf. with Eq. (13) in I]

$$
\bar{v}_{\mathrm{Hxc}}[N]=\sum_{K} \frac{U(K)}{\pi} \arctan \left(\frac{N-K}{W(K)}\right)
$$

where the $U$ 's are given in Eq. (19). In Eq. (21) we have chosen the smearing parameter $W(K)=0.16 \gamma(K) / U(K)$ where $\gamma(K)=\gamma$ is independent of $K$ since the broadening of the Coulomb blockade peaks observed in W. Liang et al. (PRL 88, 126801 (2002)) is approximately the same for all peaks (see below). We study the addition and removal of up to 6 electrons from the charge neutral SWNT due to changes of the gate $v$. In order to avoid finite-size effects we considered 22 consecutive single-particle levels $\epsilon_{0 l \nu}$. This ensures that for $16 \leq N \leq 28\left(N_{0}=22\right)$ the KS and TDDFT conductances are converged. The broadening of these levels (due to the contacts) is described by the broadening (or hybridization) matrix

$$
\left[\Gamma_{\alpha}\right]_{l \nu, l^{\prime} \nu^{\prime}}=2 \pi \sum_{k} T_{k \alpha, l \nu} \delta\left(\omega-\epsilon_{k \alpha}\right) T_{k \alpha, l^{\prime} \nu^{\prime}}
$$

where $T_{k \alpha, l \nu}=\left\langle\phi_{k \alpha}\left|H_{\text {tot }}\right| \phi_{l \nu}\right\rangle$ is the matrix element of the lead-SWNT-lead Hamiltonian $H_{\text {tot }}$ between a bulk eigenstate $\phi_{k \alpha}$ with energy $\epsilon_{k \alpha}$ of lead $\alpha=L, R$ and the eigenstate $\phi_{l \nu}$ of the SWNT. We discard the off-diagonal matrix elements of the broadening matrix since in the CB regime transport is dominated by a single resonance and interference effects (accounted for by the off-diagonal part of $\Gamma_{\alpha}$ ) can be neglected. Furthemore, we take the diagonal matrix elements of $\Gamma_{\alpha}$ all the same, which is consistent with the observation below Eq. (21). Thus

$$
\left[\Gamma_{\alpha}\right]_{l \nu, l^{\prime} \nu^{\prime}}=\delta_{l l^{\prime}} \delta_{\nu \nu^{\prime}} \gamma_{\alpha}
$$

with $\gamma_{L}=\gamma_{R} \equiv \gamma / 2$. We solve the self-consistent equation

$$
N=2 \int \frac{d \omega}{2 \pi} f(\omega) \operatorname{Tr}\left[A_{s}(\omega)\right]
$$

with the KS spectral function

$$
\left[A_{s}(\omega)\right]_{l \nu, l^{\prime} \nu^{\prime}}=\delta_{l l^{\prime}} \delta_{\nu \nu^{\prime}} \frac{\gamma}{\left(\omega-\epsilon_{0 l \nu}-\bar{v}_{\mathrm{Hxc}}[N]-v\right)^{2}+\gamma^{2} / 4}
$$

for temperatures $T<\gamma$. In Fig. $\left[\right.$ we plot $\bar{v}_{\mathrm{Hxc}}[N]$ as a function of $N$ as well as the self-consistent value of $N$ as a function of $v_{g}=v_{0}+\alpha v$ for two different values of $\gamma$. Here $v_{0}$ is chosen in order to have the same reference energy as in W. Liang et al. (PRL 88, 126801 (2002)) and we estimated the ratio $\alpha=C / C_{g} \simeq 250$ between the total capacitance and the gate capacitance from the experimental data. For a given $v$ we calculate $A_{s}$ with $N=N[v]$ and then the KS conductance from

$$
G_{s}=-\frac{\gamma}{2} \int \frac{d \omega}{2 \pi} f^{\prime}(\omega) \operatorname{Tr}\left[A_{s}(\omega)\right] .
$$

Subsequently we calculate $\partial \bar{v}_{\mathrm{Hxc}} / \partial N$ and correct $G_{s}$ according to

$$
\frac{G}{G_{s}}=\frac{2}{1+|\delta N|} \frac{1}{1+\frac{\gamma}{\gamma_{L} \gamma_{R}} G_{s} \frac{\partial \bar{v}_{\mathrm{H} \times c}}{\partial N}},
$$

see Eq. (12) of I. In Eq. (27) the quantity $\delta N$ is the deviation of the number of electrons of the HOMO from its half-filling value 1. In formulas $\delta N$ can be written as the difference between $(N-\operatorname{Int}[N])$ and 1 . In Fig. [6 we show $G_{s}$ and $G$ versus $v_{g}$ for different values of the broadening parameter $\gamma$. The value $\gamma=1.8 \mathrm{meV}$ is the one employed in Fig.4 of I. For small $\gamma$ we clearly see a pattern similar to that of Fig. 3 in I: if $N$ is close to an odd number then $G_{s}$ exhibits a Kondo plateau. The dynamical xc correction of Eq. (27) suppresses this plateau and yields the correct CB pattern. 

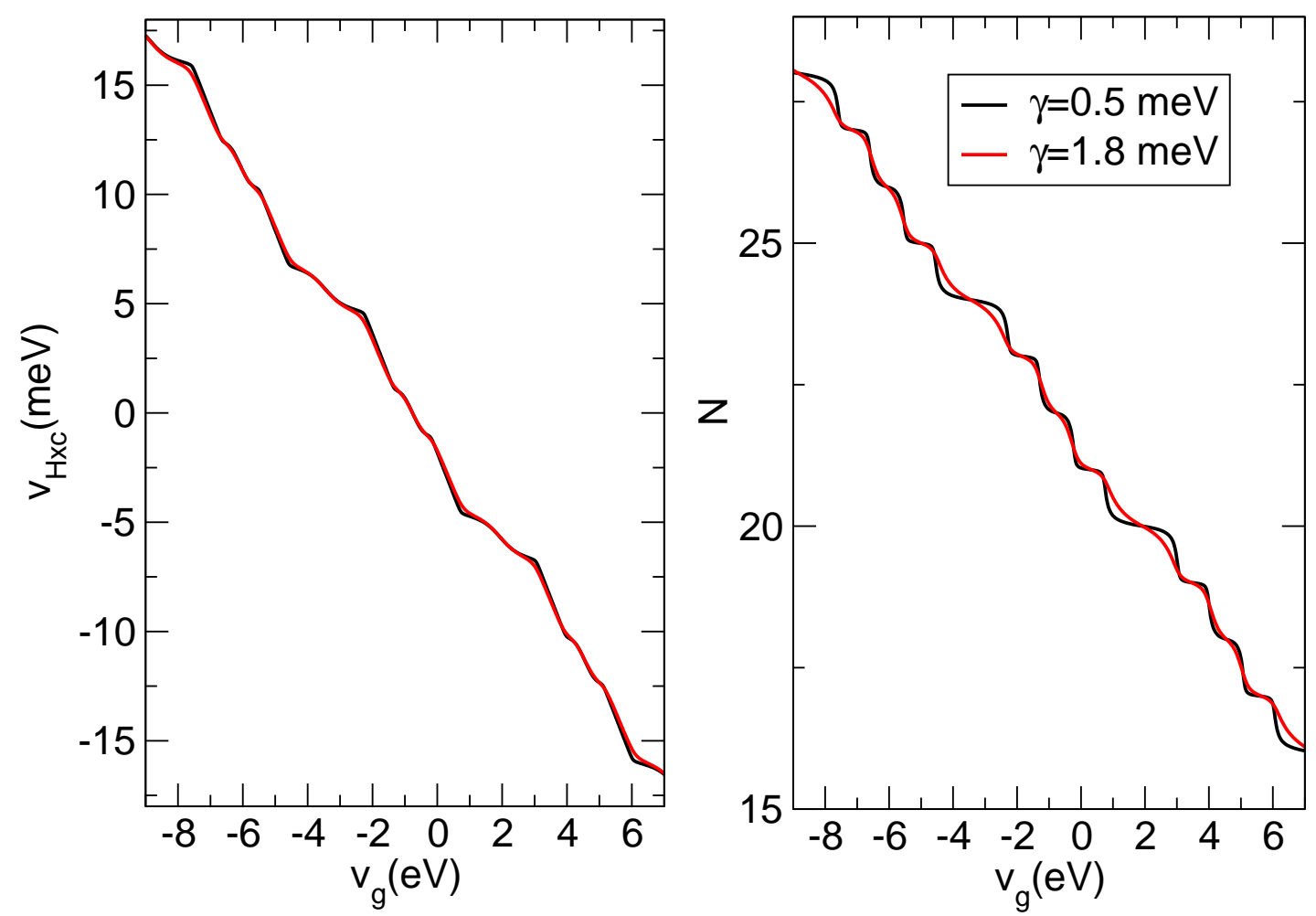

FIG. 5. KS Hxc potential (Eq. (21)) and number of electrons on the SWNT quantum dot for different values of the coupling $\gamma$ to the leads.

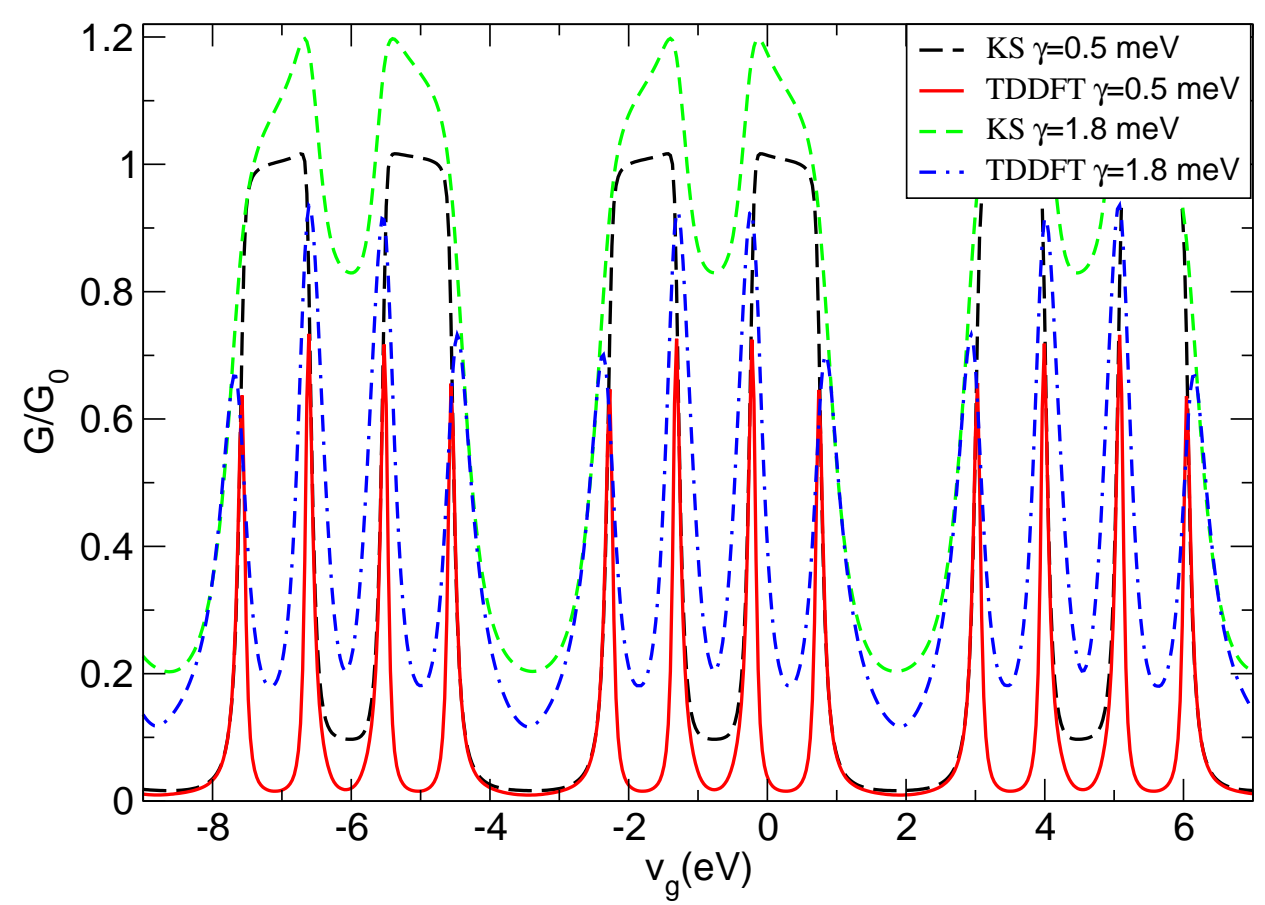

FIG. 6. KS and TDDFT corrected conductances for the SWNT quantum dot for different values of the coupling $\gamma$ to the leads. 